\title{
Оценка вклада поверхностной рекомбинации в микродисковых лазерах с помощью высокочастотной модуляции
}

\author{
(C) А.Е. Жуков ${ }^{1,2}$, Э.И. Моисеев ${ }^{1}$, Н.В. Крыжановская ${ }^{1,2}$, С.А. Блохин ${ }^{3,9}$, М.М. Кулагина ${ }^{3}$, \\ Ю.А. Гусева ${ }^{3}$, С.А. Минтаиров ${ }^{3,1}$, Н.А. Калюжный ${ }^{3,1}$, А.М. Можаров ${ }^{1}$, \\ Ф.И. Зубов ${ }^{1}$, М.В. Максимов ${ }^{1}$ \\ ${ }^{1}$ Санкт-Петербургский национальный исследовательский Академический университет \\ Российской академии наук, \\ 194021 Санкт-Петербург, Россия \\ ${ }^{2}$ Санкт-Петербургский политехнический университет Петра Великого, \\ 195251 Санкт-Петербург, Россия \\ ${ }^{3}$ Физико-технический институт им. А.Ф. Иоффре Российской академии наук, \\ 194021 Санкт-Петербург, Россия \\ E-mail: zhukale@gmail.com
}

Поступила в Редакцию 11 марта 2019 г.

В окончательной редакции 18 марта 2019 г.

Принята к публикации 18 марта 2019 г.

Исследованы микродисковые лазеры диаметром 10-30 мкм, работающие при комнатной температуре без термостабилизации, с активной областью на основе наноструктур гибридной размерности — квантовых ям-точек. Выполнены высокочастотные измерения отклика микролазеров в режиме прямой малосигнальной модуляции, с помощью которых установлены параметры быстродействия и проведен их анализ в зависимости от диаметра микролазера. Обнаружено, что $K$-фактор составляет $(0.8 \pm 0.2)$ нс, что соответствует оптическим потерям $\sim 6 \mathrm{~cm}^{-1}$, и при этом не наблюдается регулярная зависимость от диаметра. Обнаружено, что низкочастотная компонента коэффициента затухания релаксационных колебаний обратно пропорциональна диаметру. Такой характер зависимости свидетельствует об уменьшении времени жизни носителей заряда в микрорезонаторах малого диаметра, что может быть связано с преобладанием в них безызлучательной рекомбинации на боковых стенках.

Ключевые слова: микрорезонатор, микролазер, поверхностная рекомбинация, высокочастотная модуляция.

DOI: 10.21883/FTP.2019.08.48005.9098

\section{1. Введение}

Полупроводниковые микродисковые лазеры привлекают значительное внимание в качестве источников излучения для систем оптической связи, существенно более простых в изготовлении по сравнению с вертикальноизлучающими лазерами. Эпитаксиальная гетероструктура, используемая для изготовления микродискового лазера $[1,2]$, близка к применяемой для создания торцевых лазеров традиционной конструкции, поскольку высокая добротность при малых размерах резонатора достигается в микродисковом лазере не за счет толстых распределенных брэгговских отражателей, а в результате отражения света от боковых граней. В связи с малыми латеральными размерами в микродисковых лазерах важную роль приобретает проблема безызлучательной рекомбинации носителей заряда на боковых стенках резонатора, формируемых, как правило, глубоким травлением сквозь волновод и активную область. Первые инжекционные микродисковые лазеры [3] были созданы на основе материалов InGaAs/InGaAsP/InP, характеризующихся низким темпом поверхностной рекомбинации. Однако малые разрывы энергетических зон, которые достижимы в гетероструктурах на основе InP, делают такие микролазеры температурно-чувствительными [4], требуют применения принудительного охлаждения прибора, что приводит к дополнительному энергопотреблению, увеличивает размер прибора и ограничивает сферу его применения.

Альтернативой материалам, синтезируемым на подложках InP, являются гетероструктуры $\mathrm{AlGaAs} / \mathrm{GaAs}$ на подложках GaAs с квантовыми точками (In, Ga)As в активной области. Хотя эта система материалов и характеризуется более высокой скоростью поверхностной рекомбинации, применение самоорганизующихся квантовых точек (КТ), формируемых по механизму Странского-Крастанова, может до некоторой степени подавить ее влияние благодаря малой длине латеральной диффузии носителей заряда [5]. С помощью таких КТ была показана возможность лазерной генерации в микродисковых лазерах в непрерывном режиме без принудительного охлаждения вплоть до $100^{\circ} \mathrm{C}[2]$. В то же время, КТ Странского-Крастанова вследствие низкой поверхностной плотности характеризуются малым оптическим усилением, что затрудняет оптимизацию приборных параметров.

Недавно нами был предложен и реализован новый тип наноструктур, формируемых при осаждении InGaAs c относительно небольшим содержанием индия (30-50\%) на слабо разориентированную подложку $\mathrm{GaAs}(100)$ [6]. 
Они представляют собой сверхплотный массив наноостровков, названных нами „квантовые ямы-точки“ (КЯТ), поскольку сочетают некоторые особенности как квантовых точек (локализацию носителей заряда в пространстве), так и квантовых ям (высокое значение оптического усиления). С использованием КЯТ были достигнуты наибольшая на сегодня температура лазерной генерации в микродисковых лазерах в непрерывном режиме $\left(110^{\circ} \mathrm{C}\right)$, а также рекордные значения оптической мощности и коэффициента полезного действия [7]. Была показана возможность прямой модуляции с частотой по крайней мере 6 ГГц, а минимальная энергия переключения составила 1.5 пДж/бит [8]. Невыясненными, однако, остаются вопросы величины оптических потерь и влияния безызлучательной поверхностной рекомбинации на характеристики микродисковых лазеров на основе КЯТ.

В настоящей работе мы впервые применительно к микродисковым лазерам с активной областью на основе квантовых ям-точек выполнили оценки поверхностной рекомбинации и оптических потерь в таких микролазерах с помощью измерения параметров, определяющих характеристики микролазеров в режиме прямой модуляции.

\section{2. Особенности эксперимента}

Лазерная гетероструктура была синтезирована методом газофазной эпитаксии из металлоорганических соединений в реакторе низкого давления, с использованием водорода в качестве газа-носителя, а в качестве прекурсоров использовались арсин и триметилы галлия, алюминия и индия. Подложка $n^{+}$-GaAs была разориентирована на $6^{\circ}$ относительно плоскости (100). Эмиттерные слои $\mathrm{Al}_{0.34} \mathrm{Ga}_{0.66} \mathrm{As}$ ограничивали волноводный слой GaAs толщиной 0.8 мкм, в середину которого было помещено 5 рядов КЯТ, каждый из которых был сформирован осаждением 8 монослоев $\operatorname{In}_{0.4} \mathrm{Ga}_{0.6} \mathrm{As}$, отделенных друг от друга прослойками GaAs толщиной 40 нм. Лазерные микродисковые резонаторы были изготовлены с помощью фотолитографии и глубокого плазмохимического травления. Диаметр резонатора варьировался от 10.5 до 31 мкм. Верхние омические контакты к $p^{+}$-GaAs были сформированы с помощью металлизации $\mathrm{AgMn} / \mathrm{NiAu}$ и имели круглую форму. Нижний сплошной контакт к $n^{+}$-подложке был сформирован с помощью металлизации $\mathrm{AuGe} / \mathrm{Ni} / \mathrm{Au}$. Микродиски были планаризованы с помощью эпоксидного резиста SU-8 для формирования контактных площадок.

Группа из 12 микродисков различного диаметра с общим нижним контактом была напаяна на держатель, имеющий контактные площадки для подключения высокочастотного зонда. К верхней контактной площадке исследуемого микролазера индивидуально припаивалась золотая проволока диаметром 50 мкм. Микрофотография показана на рис. 1. Высокочастотные измерения проводились в диапазоне до 20 ГГц с использованием

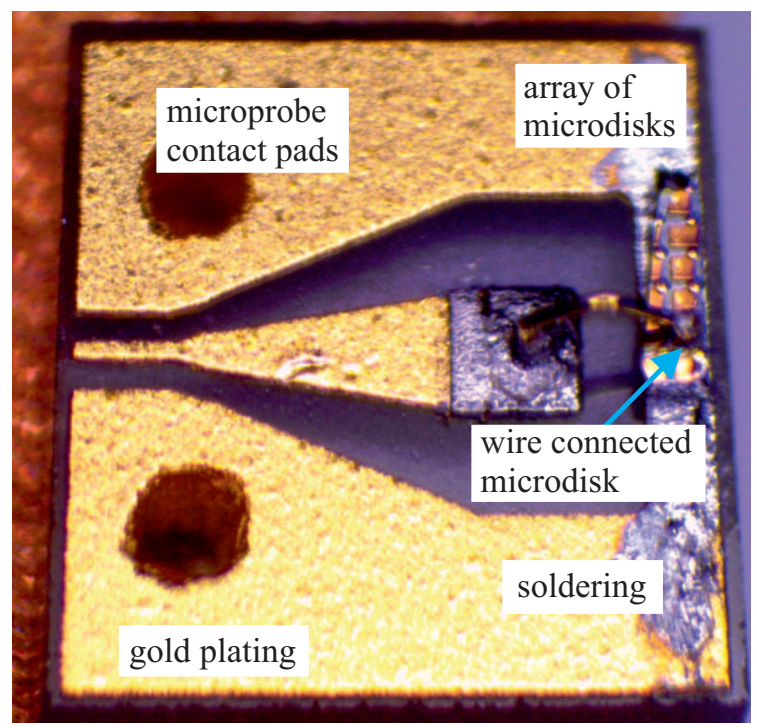

Рис. 1. Микрофотография массива микродисковых лазеров на держателе.

фотодетектора New Focus 1434 и анализатора цепей Agilent E8364В. Для каждого микролазера модуляционные кривые были записаны вплоть до тока смещения, в 3 раза превышающего пороговый ток лазерной генерации. Измерения проводили при комнатной температуре без использования температурной стабилизации и каких-либо устройств принудительного охлаждения.

\section{3. Результаты и обсуждение}

Пример амплитудно-частотного отклика $A(f)$ микродискового лазера диаметром 23 мкм при прямой малосигнальной модуляции приведен на рис. 2. Полученные экспериментальные амплитудно-частотные характеристики (АЧХ) были затем аппроксимированы с помощью выражения, которое может быть получено из анализа скоростных уравнений лазера [9],

$$
A(f)=\frac{f_{R}^{4}}{\left(f_{R}^{2}-f^{2}\right)^{2}+f^{2}(\gamma / 2 \pi)^{2}} \frac{1}{1+f^{2} / f_{C}^{2}},
$$

что позволило для каждого микролазера и определенного тока смещения определить параметры, влияющие на быстродействие, - частоту $\left(f_{R}\right)$ и коэффициент затухания $(\gamma)$ релаксационных колебаний, показанные на pис. 3 , а также паразитную частоту отсечки $\left(f_{C}\right)$.

При токах смещения $I$, не слишком близких к порогу генерации, взаимосвязь между частотой релаксационных колебаний и коэффициентом их затухания описывается выражением [10]

$$
\gamma=K f_{R}^{2}+\gamma_{0},
$$

где $K$ - так называемый $K$-фактор, зависящий от оптических потерь в резонаторе и коэффициента нелинейного насыщения усиления активной области, $\gamma_{0}-$ 
доминирующая при малых токах смещения (низкочастотная) компонента коэффициента затухания (так называемое смещение коэффициента затухания). На вставке к рис. 3 представлен пример такой зависимости для случая микролазера диаметром 27 мкм. Каждая точка представляет собой пару $f_{R}^{2}$ и $\gamma$, значения которых были определены для некоторого тока смещения. Аппроксимация с помощью выражения (2) дает для этого микролазера $K=(0.75 \pm 0.05)$ нс и $\gamma_{0}=(2.5 \pm 0.5)$ ГГц (наблюдаемое в области малых частот отклонение от линейной зависимости обусловлено вкладом спонтанного излучения в лазерную моду [9], который становится пренебрежимо малым по мере роста тока смещения).

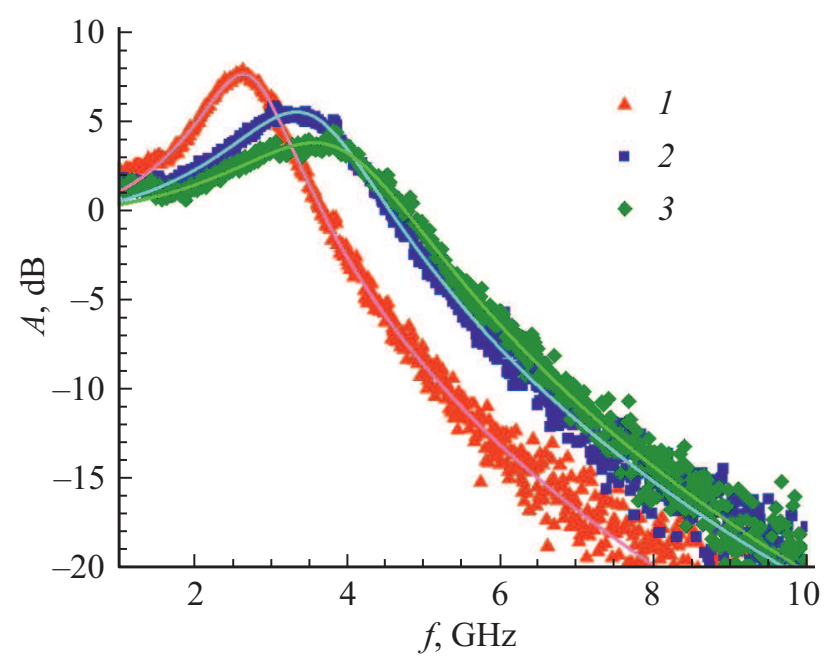

Рис. 2. Малосигнальные модуляционные кривые для микродискового лазера диаметром 23 мкм при постоянном токе смещения, мА: $1-13,2-16,3-19$. Точки - эксперимент, линии - аппроксимация выражением (1).

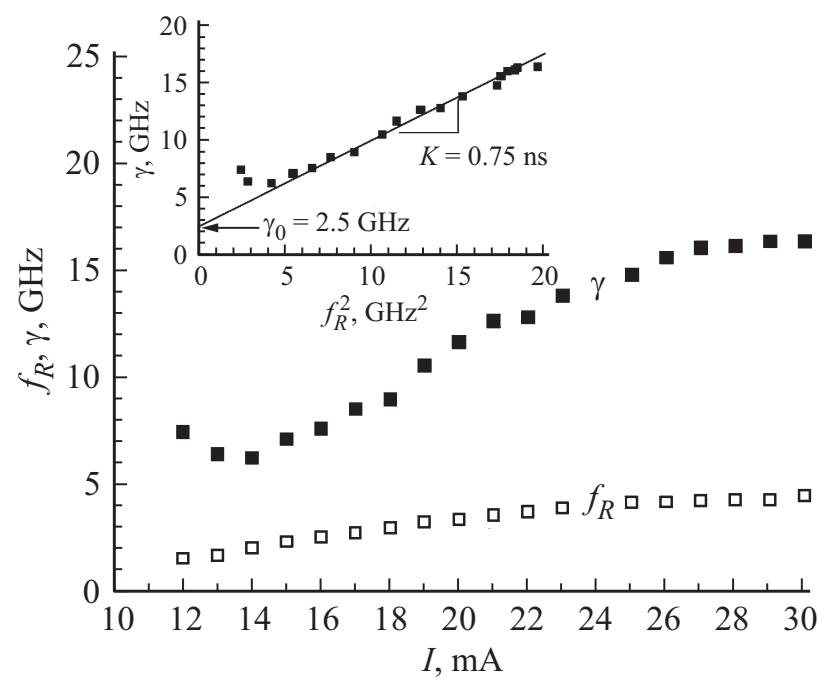

Рис. 3. Частота и коэффициент затухания релаксационных колебаний в зависимости от тока смещения для микролазера диаметром 27 мкм. На вставке - взаимосвязь между частотой и коэффициентом затухания релаксационных колебаний (точки) и аппроксимация выражением (2) (линия).

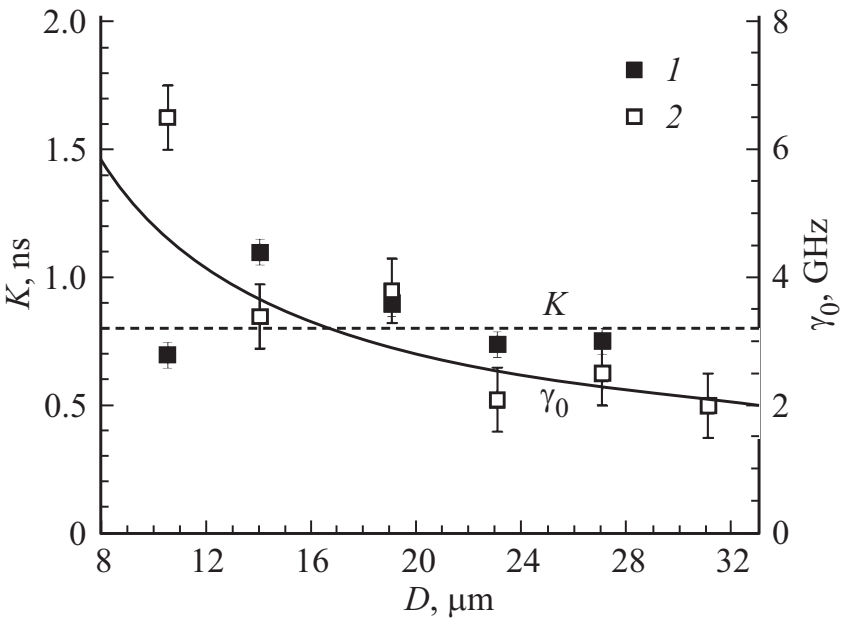

Рис. 4. Значения $K$-фактора (точки 1 ) и низкочастотной компоненты коэффициента затухания релаксационных колебаний $\gamma_{0}$ (точки 2) в зависимости от диаметра микролазеров. Штриховая линия $-K=0.8$ нс, сплошная линия - аппроксимация выражением (4).

Рис. 4 обобщает значения $K$-фактора, определенные для микродисковых лазеров различного диаметра. Как видно, отсутствует какая-либо регулярная зависимость $K$-фактора от диаметра микролазера, а это означает, что и оптические потери не зависят от диаметра в исследованном диапазоне размеров. Это согласуется с предсказаниями теории [11], согласно которым экспоненциально зависящие от диаметра потери на вывод излучения из микродиска или микрокольца, обусловленные кривизной резонатора, становятся заметными, лишь когда его размер сопоставим с длиной волны излучения. Оптические потери, вызванные рассеянием света на шероховатостях боковых стенок, обратно пропорциональны объему микродиска [12], так что значения $K$-фактора должны уменьшаться при уменьшении диаметра, если прочие параметры микрорезонатора (прежде всего характерный размер рассеивающих центров на поверхности) остаются неизменными. Очевидно, что в реальности микрошероховатости, возникающие в процессе изготовления лазерного микрорезонатора, варьируются от процесса к процессу, что и приводит к разбросу значения оптических потерь и, как результат, нерегулярному поведению $K$-фактора. Его среднее значение (штриховая линия на рис. 4) составляет $\sim(0.8 \pm 0.2)$ нс, что соответствует предельной частоте модуляции $\sim 11$ ГГц.

То обстоятельство, что $K$-фактор не зависит от диаметра микролазера, означает неизменность оптических потерь, а значит, все исследуемые микролазеры должны характеризоваться близкими значениями пороговой концентрации носителей заряда. Ранее мы показали, что квадрат частоты релаксационных колебаний линейно изменяется с выходной мощностью излучения микролазера по крайней мере вплоть до 4ГГц [8], что позволяет пренебречь в этом диапазоне токов смеще- 
ния влиянием нелинейного насыщения усиления. Это в свою очередь позволяет с использованием выражения $K \approx 4 \pi^{2}\left(v_{\mathrm{gr}} \alpha\right)^{-1}$ оценить величину оптических потерь в исследуемых микролазерах как $(6 \pm 2) \mathrm{cm}^{-1}$ (при расчете групповой скорости фотонов лазерной моды $v_{\mathrm{gr}}$ полагали величину эффективного модового индекса равной 3.5). Такое значение оптических потерь заметно меньше величины насыщенного оптического усиления использованной активной области $\left(>50 \mathrm{~cm}^{-1}\right)$, так что концентрация носителей должна быть близка к той, при которой происходит инверсия заселенности квантовых точек.

При анализе параметров, полученных с помощью анализа малосигнальной прямой модуляции, наибольший интерес, как правило, представляют значения $K$-фактора, поскольку они определяют предельное быстродействие лазера, достигаемое при сильном демпфировании релаксационных колебаний [13]. Однако в настоящей работе нас также интересуют и значения $\gamma_{0}$, которые в большинстве экспериментальных работ не обсуждаются. В связи с этим следует отметить, что смещение коэффициента затухания релаксационных колебаний $\gamma_{0}$, как следует из анализа скоростных уравнений [14], обусловлено конечным временем жизни $\tau$ носителей заряда в активной области, т.е. темпом рекомбинации:

$$
\gamma_{0}=\frac{1}{\chi \tau} \approx \frac{1}{\tau}
$$

где $\chi$ - типично близкий к 1 фактор, зависящий от транспорта носителей через волновод и их захвата в активную область [15]. Таким образом, анализ $\gamma_{0}$ позволит определить особенности рекомбинации носителей заряда в различных микролазерах (в (3) мы пренебрегаем членом, связанным с вкладом спонтанного излучения в лазерную моду, поскольку, как отмечалось выше, подгонка экспериментальных данных с помощью (2) осуществляется вдали от порога лазерной генерации).

На рис. 4 приведены значения $\gamma_{0}$ в зависимости от диаметра $D$. Несмотря на заметный разброс, отчетливо прослеживается тенденция к увеличению $\gamma_{0}$ при снижении размера микролазера, т.е. к уменьшению времени жизни носителей. Экспериментальная зависимость может быть аппроксимирована выражением (сплошная линия на рис. 4)

$$
\gamma_{0}=\frac{a}{D}+\frac{1}{\tau_{0}},
$$

где коэффициент $a \approx(40 \pm 10)$ ГГц $\cdot$ мкм $\tau_{0} \approx(1.2 \pm 0.3)$ нс.

Время жизни носителей заряда (более точно, дифференциальное время жизни) определяется зависимостью темпа рекомбинации $R$ от концентрации $n$ :

$$
\tau^{-1}=\frac{d R(n)}{d n}
$$

Поскольку, как мы установили, концентрация носителей заряда во всех микродисках, независимо от их диаметра,

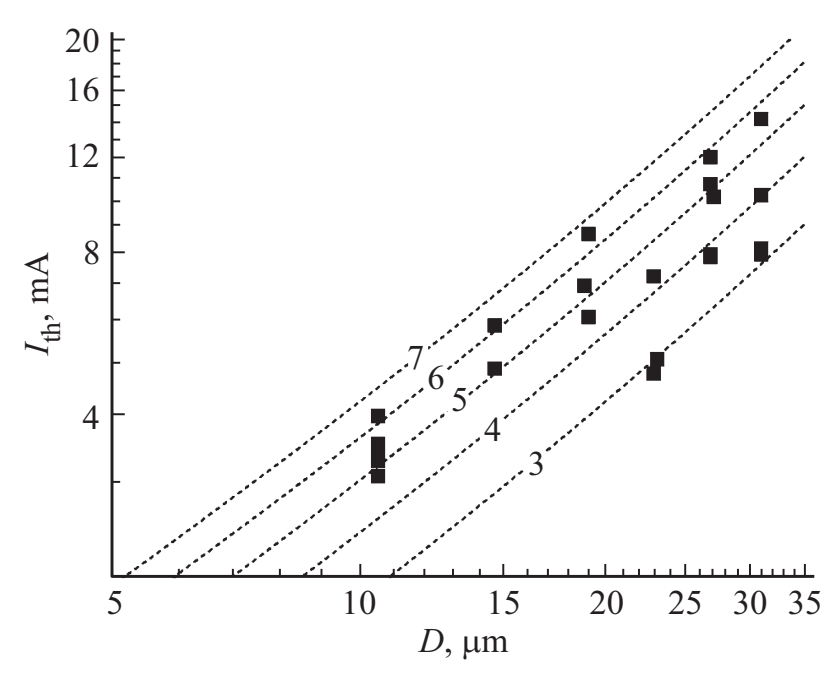

Рис. 5. Зависимости порогового тока микродисковых лазеров от диаметра: точки - эксперимент, линии - аппроксимация выражением (8). Указаны значения пороговой концентрации в единицах $10^{12} \mathrm{~cm}^{-2}\left(3 \cdot 10^{12}-7 \cdot 10^{12} \mathrm{~cm}^{-2}\right)$.

остается приблизительно постоянной, величина $\tau$ должна оставаться постоянной, если только сам темп рекомбинации не зависит от диаметра в явном виде. Все механизмы объемной рекомбинации, как излучательные, так и безызлучательные, протекающие как непосредственно в активной области, так и в волноводе, не содержат такой зависимости. В отличие от них темп безызлучательной поверхностной рекомбинации $R_{s r}$, возникающей на боковых стенках микрорезонатора, в явном виде определяется отношением периметра $(P=\pi D)$ активной области к ее площади $\left(S=\pi D^{2} / 4\right)[9]$ :

$$
R_{s r}=\frac{P}{S} v_{s} n \propto \frac{1}{D},
$$

где $v_{s}$ - скорость поверхностной рекомбинации.

Комбинируя (3)-(6), мы получаем следующее выражение для смещения коэффициента затухания, определяемого безызлучательной поверхностной рекомбинацией:

$$
\gamma_{0 s r}=\frac{P}{S} v_{s}=\frac{4 v_{s}}{D}
$$

Полученное выражение позволяет нам сопоставить подгоночный параметр $a$, с учетом множителя 4, со скоростью поверхностной рекомбинации. При этом параметр $\tau_{0}$, очевидно, имеет смысл времени жизни носителей заряда, определяемого механизмами рекомбинации, протекающими в объеме.

На рис. 5 представлены экспериментальные значения порога генерации в зависимости от диаметра микродискового лазера (приведены результаты для всей имеющейся совокупности данных для аналогичных микролазеров, а не только для тех структур, которые были исследованы в режиме прямой модуляции). В области 
малых диаметров наблюдается тенденция к росту значения порогового тока пропорционально первой степени диаметра микродиска. Иными словами, пороговая плотность тока, определяемая как $I_{\mathrm{th}} / S$, не остается постоянной, но увеличивается при уменьшении $D$.

Пороговый ток $I_{\text {th }}$ микролазера можно представить суммой двух слагаемых:

$$
I_{\mathrm{th}}=S j_{\mathrm{th} 0}+e P v_{s} n_{\mathrm{th}},
$$

из которых первое, $\propto D^{2}$, соответствует рекомбинации в объеме и характеризуется постоянной плотностью тока рекомбинации $j_{\mathrm{th} 0}=e n_{\mathrm{th}} / \tau_{0}$, а второе слагаемое, $\propto D$, описывает вклад поверхностной рекомбинации [9]; $n_{\text {th }}$ имеет смысл двумерной концентрации носителей на пороге генерации. Результаты расчета порогового тока в микродисковых лазерах разного диаметра с использованием выражения (8) приведены на рис. 5. Параметры, характеризующие поверхностную и объемную рекомбинации, взяты в соответствии с обсуждавшимися выше значениями, $n_{\mathrm{th}}$ варьируется в диапазоне $(3-7) \cdot 10^{12} \mathrm{~cm}^{-2}$. Как видно, наиболее близко всю совокупность экспериментальных данных характеризует значение пороговой концентрации $\sim 5 \cdot 10^{12} \mathrm{~cm}^{-2}$. Эта оценка по порядку величины согласуется с концентрацией $\left(\sim 2.5 \cdot 10^{12} \mathrm{~cm}^{-2}\right)$ носителей заряда в активной области, необходимой для достижения инверсии заселенности, с учетом числа рядов КЯТ (5) и поверхностной плотности $\left(\sim 0.5 \cdot 10^{12} \mathrm{~cm}^{-2}\right)$ островков в каждом ряду, на порядок превышающей значения, характерные для КТ, формируемых по механизму Странского-Крастанова. Отметим, что для наиболее низкопороговых исследованных микролазеров оценка концентрации носителей заряда дает величину $\sim 3 \cdot 10^{12} \mathrm{~cm}^{-2}$.

Выражения (4) и (8) предсказывают, что в области больших диаметров доминирует объемная рекомбинация, а при малых размерах микродискового лазера поверхностная безызлучательная рекомбинация. Критический размер микродиска, при котором последняя начинает преобладать, $D_{\mathrm{cr}}=\alpha \tau_{0}$, составляет 50 мкм с учетом извлеченных из эксперимента значений параметров. Это означает, что для всей исследуемой совокупности микролазеров вклад поверхностной рекомбинации является существенным.

\section{4. Заключение}

Таким образом, нами исследованы параметры быстродействия микродисковых лазеров с активной областью, представляющей собой сверхплотный массив квантовых ям-точек, формируемых на вицинальных подложках. Определенное с помощью $K$-фактора значение оптических потерь относительно невелико и составляет $\sim 6 \mathrm{~cm}^{-1}$ независимо от диаметра микродиска. В этих условиях экспериментально наблюдаемый при уменьшении диаметра рост величины низкочастотной компоненты коэффициента затухания релаксационных колебаний, определяемой временем жизни носителей заряда в активной области, может быть объяснен соответствующим увеличением темпа поверхностной рекомбинации в микродисках меньшего размера. Это согласуется с наблюдаемым возрастанием пороговой плотности тока при уменьшении диаметра. Отметим, что такое поведение не характерно для инжекционных микролазеров на основе КТ, сформированных по механизму Странского-Крастанова [16], для которых в широком диапазоне значений диаметра наблюдается постоянное значение пороговой плотности тока. Отличие может быть вызвано меньшей энергией локализации носителей заряда в КЯТ и, как результат, в более свободной латеральной миграции носителей вдоль плоскости активной области.

\section{Финансирование работы}

Работа выполнена при поддержке проекта РНФ 18-12-00287. Планаризация микродисков (А.М.М.) была разработана и выполнена при поддержке Министерства образования и науки России (3.9796.2017/8.9 и 16.2593.2017/4.6).

\section{Конфликт интересов}

Авторы заявляют, что у них нет конфликта интересов.

\section{Список литературы}

[1] M.-H. Mao, H.-C. Chien, J.-Z. Hong, C.-Y. Cheng. Opt. Express, 19, 14145 (2011).

[2] N.V. Kryzhanovskaya, E.I. Moiseev, Yu.V. Kudashova, F.I. Zubov, A.A. Lipovskii, M.M. Kulagina, S.I. Troshkov, Yu.M. Zadiranov, D.A. Livshits, M.V. Maximov, A.E. Zhukov. Electron. Lett., 51, 1354 (2015).

[3] A.F.J. Levi, R.E. Slusher, S.L. Mc Call, T. Tanbun-Ek, D.L. Coblentz, S.J. Pearton. Electron. Lett., 28, 1011 (1992).

[4] A.K. Sokol, R.P. Sarzala. Optica Applicata, 43, 325 (2013).

[5] A. Fiore, M. Rossetti, B. Alloing, C. Paranthoen, J.X. Chen, L. Geelhaar, H. Riechert. Phys. Rev. B, 70, 205311 (2004).

[6] S.A. Mintairov, N.A. Kalyuzhnyy, V.M. Lantratov, M.V. Maximov, A.M. Nadtochiy, S. Rouvimov, A.E. Zhukov. Nanotechnology, 26, 385202 (2015).

[7] E. Moiseev, N. Kryzhanovskaya, M. Maximov, F. Zubov, A. Nadtochiy, M. Kulagina, Yu. Zadiranov, N. Kalyuzhnyy, S. Mintairov, A. Zhukov. Optics. Lett., 43, 4554 (2018).

[8] A.E. Zhukov, N.V. Kryzhanovskaya, M.V. Maximov. Int. Conf. Frontiers of 21st Century Physics and Ioffe Institute“ (St. Petersburg, Russia, 2018) p. 33.

[9] L.A. Coldren, S.W. Corzine, M.L. Masanovic. Diode Lasers and Photonic Integrated Circuits, 2nd edn (N.Y., Wiley, 2012).

[10] R. Nagarajan, T. Fukushima, M. Ishikawa, J.E. Bowers, R.S. Geels, L.A. Coldren. IEEE Photon. Technol. Lett., 4, 121 (1992).

[11] R.E. Slusher, A.F.J. Levi, U. Mohideen, S.L. Mc Call, S.J. Pearton, R.A. Logan. Appl. Phys. Lett., 63, 1310 (1993).

[12] M. Borselli, T.J. Johnson, O. Painter. Opt. Express, 13, 1515 (2005). 
[13] E. Kapon. Semiconductor Lasers I: Fundamentals (San Diego, Academic Press, 1999).

[14] R. Nagarajan, M. Ishikawa, T. Fukushima, R.S. Geels, J.E. Bowers. IEEE J. Quant. Electron., 28, 1990 (1992).

[15] H. Su, L.F. Lester. J. Phys. D: Appl. Phys., 38, 2112 (2005).

[16] Н.В. Крыжановская, М.В. Максимов, С.А. Блохин, М.А. Бобров, М.М. Кулагина, С.И. Трошков, Ю.М. Задиранов, А.А. Липовский, Э.И. Моисеев, Ю.В. Кудашова, Д.А. Лившиц, В.М. Устинов, А.Е. Жуков. ФТП, 50, 393 (2016).

Редактор Л.В. Шаронова

\section{Estimation of the surface recombination impact in microdisk lasers by means of high-frequency modulation technique}

A.E. Zhukov ${ }^{\mathbf{1}, 2}$, E.I. Moiseev ${ }^{1}$, N.V. Kryzhanovskaya ${ }^{\mathbf{1 , 2}}$, S.A. Blokhin ${ }^{3}$, M.M. Kulagina ${ }^{3}$, Yu.A. Guseva ${ }^{3}$, S.A. Mintairov ${ }^{3,1}$, N.A. Kalyuzhnyy ${ }^{3,1}$, A.M. Mozharov ${ }^{1}$, F.I. Zubov' ${ }^{1}$, M.V. Maximov ${ }^{1}$

${ }^{1}$ St. Petersburg National Research Academic University, Russian Academy of Sciences, 194021 St. Petersburg, Russia

${ }^{2}$ Peter the Great Polytechnic University, 195251 St. Petersburg, Russia

${ }^{3}$ loffe institute,

194021 St. Petersburg, Russia

Abstract Microdisk lasers with a diameter of $10-30 \mu \mathrm{m}$, operating at room temperature without thermal stabilization, with an active region based on nanostructures of hybrid dimensionality (quantum well-dots) are studied. Measurements of the highfrequency small-signal modulation response of the microlasers were carried out for evaluation of the dynamic characteristics and their analysis as a function of the microlaser diameter. It was found that the $K$-factor is $(0.8 \pm 0.2) \mathrm{ns}$, which corresponds to an optical loss of $\sim 6 \mathrm{~cm}^{-1}$; it does not demonstrate a regular dependence on the diameter. It was found that the damping factor offset of the relaxation oscillations is inversely proportional to the diameter. This character of dependence indicates a decrease in the lifetime of charge carriers in microresonators of smaller diameters and may be attributed to the predominance of nonradiative recombination at the sidewalls. 